\title{
An industry update: the latest developments in therapeutic delivery covering August 2019
}

\author{
Oliver C Steinbach*,1 \\ ${ }^{1}$ Trans Code Therapeutics Inc., 6 Liberty Square \#2382, Boston, MA 02109, USA \\ *Author for correspondence: Tel.: +1 216307 7964; oliver.steinbach@transcodetherapeutics.com
}

First draft submitted: 27 November 2019; Accepted for publication: 2 December 2019; Published online: 18 December 2019

Keywords: microparticles • nanotechnology • ophthalmology • RNAi

The present industry update covers the period 1-31 August 2019, with information sourced from company press releases, regulatory and patent agencies as well as scientific literature.

\section{Business development \\ Reports \\ Cancer drug delivery}

A report published in August 2019 on cancer drug delivery described the challenge of not simply reformulating existing drugs but the increasing focus on targeted delivery, complementing chemotherapy and radiotherapy to reduce adverse side effects and drug resistance. The several innovative methods currently investigated include injectable microparticles guided by magnetic field for targeting, polyethylene glycol modifications to overcome barriers, encapsulation in liposomes and conjugation to monoclonal antibodies to deliver cancer drugs such as radionucleotides, toxins and chemotherapeutic agents to the tumor. Antisense oligonucleotides and small interfering RNAs (siRNAs) appear to have overcome delivery obstacles and have seen the first product launches with many to follow, which are now in clinical trials, including those for cancer. The market value of drug-delivery technologies and oncology drugs are more and more tied to each other, and the publisher provides market estimates from 2018-2028, which are categorized by organs, clinical indications and technologies [1].

\section{Financing \\ Kala Pharmaceuticals}

Kala Pharmaceuticals, Inc. (MA, USA) is a biopharmaceutical company using its proprietary, AMPPLIFY ${ }^{\top M}$, a mucus-penetrating particle drug delivery technology that is currently applied to the treatment of eye diseases. On 15 August 2019, nonstatutory stock options were granted to new employees as inducement awards outside the company's 2017 Equity Incentive Plan. Kala has utilized the AMPPLIFY technology for corticosteroid loteprednol etabonate that led to US FDA approval in August 2018 of INVELTYS ${ }^{\circledR}$ for the treatment of inflammation and pain, following ocular surgical interventions. The company's lead candidate is KPI- $1210.25 \%$ is intended for the temporary relief of dry eye disease symptoms [2]. Kala Pharmaceuticals received on 8 August 2019 an FDA complete response letter regarding the new application of this drug. The regulators are considering that efficacy data from an additional clinical trial will be necessary to support a resubmission. Results of the ongoing STRIDE 3 Phase III clinical trial, a multicenter, randomized, double-blind, placebo-controlled, parallel-arm study comparing KPI-121 $0.25 \%$ to a vehicle (placebo) are expected by the end of 2019 and will be the basis of the response to the FDA in the first half of 2020. The STRIDE 3 trial subjects underwent a 2-week run-in period with a vehicle. The individuals who continued to meet inclusion/exclusion criteria after the run-in were randomized to receive either, KPI-121 $0.25 \%$ or a vehicle for 2 weeks [2]. 


\section{Licensing}

Ironwood Pharmaceuticals

Alnylam Pharmaceuticals, Inc. (MA, USA) is a clinical stage RNAi therapeutics company. On 13 August 2019, Ironwood Pharmaceuticals, Inc. (MA, USA) announced a nonexclusive, 3-year education and promotional agreement for Alnylam's givosiran, an investigational RNAi therapeutic that targets aminolevulinic acid synthase 1 (ALAS1) for the potential treatment of acute hepatic porphyria. Givosiran has received priority review designation and breakthrough therapy designation from the FDA as well as orphan drug designation. Ironwood will provide, under this agreement, acute hepatic porphyria disease education to gastroenterologists and other healthcare providers that are currently approached for LINZESS ${ }^{\circledR}$ (linaclotide). After approval of givosiran, Ironwood clinical sales forces will start promotional efforts that complement Alnylam's commercialization efforts. Ironwood will receive fixed payments and royalties on net sales for prescriptions or referrals. Alnylam will take ownership for all other aspects of givosiran, including all global development and commercialization rights [3].

\section{Arrowhead Pharmaceuticals}

Arrowhead Pharmaceuticals Inc. (CA, USA) announced on 28 August 2019 that Janssen Pharmaceuticals, Inc. (Beerse, Belgium) has begun dosing different combination regimens, including JNJ-3989, a liver-targeted, investigational, antiviral therapeutic for subcutaneous injection that is designed to treat chronic hepatitis B infection via the RNAi mechanism and JNJ-6379 for the treatment of chronic hepatitis B virus infection in a Phase IIb study (REEF-1). REEF-1 is a multicenter, double-blind, active-controlled, randomized study to investigate the efficacy and safety of different combination regimens. Arrowhead received a $\$ 25$ million milestone payment from Janssen at the start of the study. JNJ-3989 is an investigational, orally administered, capsid assembly, modulator which forms normal capsid structures. Arrowhead entered into a license and collaboration agreement with Janssen in October 2018 to develop and commercialize ARO-HBV [4].

\section{Regulatory news \& approvals \\ Clinical trials \\ Arrowhead Pharmaceuticals}

Also Arrowhead Pharmaceuticals Inc. announced on 8 August 2019 the first patient enrolled in its SEQUOIA (AROAAT2001) trial. This is a Phase II/III clinical study; patients with alpha-1 antitrypsin deficiency associated liver disease were subcutaneously administered ARO-AAT using a placebo-controlled, adaptive design for safety, efficacy and tolerability evaluation. ARO-AAT represents a new generation of RNAi drugs. The primary efficacy readout is the fraction of patients improving to reach a two-point histologic grading scale of alpha- 1 antitrypsin deficiency associated liver disease. In this trial, no indication of liver fibrosis worsening was observed by a biopsy taken at the end of study [5].

\section{MedinCell}

A second long-acting, injectable, antipsychotic termed $\mathrm{BEPO}{ }^{\circledR}$ technology, developed by MedinCell (Montpellier, France), is based on proprietary copolymers and a biocompatible solvent that forms a fully bioresorbable depot once injected locally. MedinCell's technology received FDA Investigational New Drug clearance on 26 August 2019 and a first-in-human study is due to begin before the end of 2019. mdc-TJK is one of three antipsychotic products in development that is based on MedinCell's technology [6].

\section{Patents \\ Imbrium Therapeutics}

Imbrium Therapeutics LP (CT, USA), an operating subsidiary of Purdue Pharma LP (CT, USA), collaborates with SpineThera, Inc. (MN, USA) and announced on 22 August 2019 the issuing of US Patent Number 10,350,222. This patent is a protecting composition of matter for SX600, which is the third US patent covering treatment methods. This is an injectable, dexamethasone acetate, micro-suspension development candidate that is reformulated into microparticles that are smaller than red blood cells, with sustained release delivery to treat back pain. Under the agreement, Purdue Pharma LP obtained an exclusive option to acquire SpineThera, Inc., including its worldwide technologies and product rights [7]. 


\section{Bioasis}

Bioasis Technnologies Inc. (CT, USA) is a preclinical research-stage biopharmaceutical company that develops a platform technology termed $\mathrm{xB}^{3^{T M}} \cdot \mathrm{xB}^{3}$ delivers drugs across the blood-brain barrier to treat central nervous system disorders, including brain cancers and neurodegenerative diseases. The company announced on 21 August 2019 that the China National Intellectual Property Administration (Beijing, China) issued a notice to undergo registration procedures and grant patent rights for a Chinese patent application. This application intends to protect Bioasis' P97 conjugates with therapeutic agents, such as $\mathrm{xB}^{3}-001$. Bioasis' lead product conjugate with trastuzumab is in development for the treatment of HER2+ breast cancer brain metastases where P97 is the carrier across the blood-brain barrier [8].

\section{Publications}

\section{CBSET}

CBSET (MA, USA) is a not-for-profit preclinical research institute dedicated to biomedical research and education. The company announced on 28 August 2019 that its scientists have published data reflecting over 20 years of NIH-funded fundamental research, addressing safety and efficacy of balloon and stent-based paclitaxel retention, in normal and pathological arteries, in many procedures every year. These devices have a three-phase local drug retention dynamic: luminal coating dissolution, tissue embedded coating dissolution and receptor dissociation respectively, with the latter determining the rate of terminal clearance [9].

\section{Reviews}

Multiple reviews on the subject of nanoplatforms for drug delivery were published this month, describing the multitude of drug-delivery platforms established to target and control delivery of therapeutic compounds in a variety of clinical indications. In the review by Singh et al., the authors discuss novel and established drug formats, such as peptides, nucleic acids and therapeutic genes, that have demonstrated potential as nanomedicines to address many chronic diseases [10]. Mansoor et al. discuss current insulin therapy for the management of diabetes and the challenges of patient compliance when taking multiple and daily insulin injections. To improve insulin therapy, novel drug-delivery systems and alternative routes of administration are investigated through advances in polymer science and nanotechnology, which are discussed in this review [11]. Onaciu et al. describe hydrogels as 3D polymeric networks, which are increasingly applied to drug delivery. Due to their porous, sol-gel structure, they can entrap considerable quantities of compounds. In addition, hydrogels have biocompatibility and biodegradability as well as controlled release properties, which qualify hydrogels as potential drug-delivery systems. This is also confirmed in in vivo studies in various disease indications. Combined with the results of clinical trials, this points toward a very encouraging and promising use of hydrogels as future targeted therapy strategies [12].

\section{Conclusion}

Multiple companies reported on their progress in the use of microparticles for drug delivery, including in capital raising (Kala Pharmaceuticals), patient protection (Imbrium Therapeutics) and clinical trials (MedinCell, Kala Pharmaceuticals). Also, RNAi therapeutics are furthered into the market via the clinic, since delivery obstacles have been increasingly overcome (Arrowhead Pharmaceuticals, Alnylam Pharmaceuticals). Multiple scientific reviews that have been published describe the progress, depth and breadth of nanotechnology, which is increasingly applied to drug-delivery challenges.

\section{Financial \& competing interests disclosure}

OC Steinbach is an employee of TransCode Therapeutics Inc. The author has no other relevant affiliations or financial involvement with any organization or entity with a financial interest in or financial conflict with the subject matter or materials discussed in the manuscript apart from those disclosed

No writing assistance was utilized in the production of this manuscript.

\section{References}

1. Drug delivery in cancer - technologies, markets \& companies. (2019).

www.researchandmarkets.com/reports/4748166/drug-delivery-in-cancer-technologies-markets?utm_source=BW \&utm_medium=PressR elease\&utm_code $=5$ trr $5 \mathrm{w} \&$ utm_campaign $=1282967+-+$ Cancer+Drug+Delivery+Research + Report $+2019 \% 3 \mathrm{a}+$ Focus + on + Technologies $\% 2 c+$ Markets+\%26+Companies+-+Updated+Estimates+to+2029\&utm_exec $=$ chdo54prd 
2. Kala pharmaceuticals reports inducement grant under Nasdaq listing rule 5635(C)(4). (2019). http://investors.kalarx.com/news-releases/news-release-details/kala-pharmaceuticals-reports-inducement-grant-under-nasdaq-8

3. Ironwood pharmaceuticals and alnylam pharmaceuticals enter U.S. GI disease education and promotional agreement for Alnylam's Givosiran in acute hepatic porphyria (AHP). (2019). https:

//investor.ironwoodpharma.com/press-releases/press-release-details/2019/Ironwood-Pharmaceuticals-and-Alnylam-Pharmaceuticals-E nter-US-GI-Disease-Education-and-Promotional-Agreement-for-Alnylams-Givosiran-in-Acute-Hepatic-Porphyria-AHP/default.aspx

4. Arrowhead collaborator Janssen begins REEF-1 Phase IIb combination study in patients with chronic hepatitis B infection. (2019). http://ir.arrowheadpharma.com/news-releases/news-release-details/arrowhead-collaborator-janssen-begins-reef-1-phase-2b

5. Arrowhead pharmaceuticals doses first patient in SEQUOIA Phase II/III study of ARO-AAT for treatment of alpha-1 liver disease. (2019). http://ir.arrowheadpharma.com/node/14736/pdf

6. Long-acting injectable antipsychotic using MedinCell's technology receives FDA IND clearance to initiate clinical activities. (2019). www.medincell.com/wp-content/uploads/2019/08/2019_08_26_MEDCL_PR_mdcTJK_IND-clearance_ENG_final.pdf

7. Imbrium Therapeutics L.P. and Spinethera announce issuance of U.S. patent covering composition of matter protection forSX600, a non-opioid product in development for epidural steroid injection for the treatment of low back pain. (2019).

www.imbriumthera.com/news/imbrium-therapeutics-1-p-and-spinethera-announce-issuance-of-u-s-patent-covering-composition-ofmatter-protection-for-sx600-a-non-opioid-product-in-development-for-epidural-steroid-injection-for-th/

8. Bioasis announces registration and grant notice of patent application in China relating to xB3 platform technology for the delivery of therapeutic agents across the blood-brain barrier. (2019).

www.biospace.com/article/releases/bioasis-announces-registration-and-grant-notice-of-patent-application-in-china-relating-to-xb3-pla tf orm-technology-for-the-delivery-of-therapeutic-agents-across-the-blood-brain-barrier/

9. Computational model of coating and tissue morphology determinants of balloon based drug retention and tissue dosing suggest a new paradigm for accelerated treatment optimization: CBSET data published in Journal of Controlled Release. (2019). http://cbset.org/press-release-computational-model-coating-tisseu-morphology-determinants-balloon-absed-drug-retention/

10. Singh AP, Biswas A, Shukla A, Maiti P. Targeted therapy in chronic diseases using nanomaterial-based drug delivery vehicles. SignalTransduct. Target. Ther. 4, 33-54 (2019).

11. Mansoor S, Kondiah PPD, Choonara YE, Pillay V. Polymer-based nanoparticle strategies for insulin delivery. Polymers (Basel) 11(9), 1380-1407 (2019).

12. Onaciu A, Munteanu RA, Moldovan AI, Moldovan CS, Berindan-Neagoe I. Hydrogels based drug delivery synthesis, characterization and administration. Pharmaceutics 11(9), 432-456 (2019). 